\title{
Detecting Correlation Changes in Electrophysiological
}

\author{
Data \\ ${\text { Jianhua } \mathrm{Wu}^{\dagger} \quad \text { Keith Kendrick }}^{\ddagger} \quad$ Jianfeng Feng $^{\dagger}$ \\ ${ }^{\dagger}$ Department of Computer science, University of Warwick CV4 7AL, UK \\ ${ }^{\ddagger}$ Laboratory of Cognitive and Behavioural Neuroscience \\ The Babraham Institute, Cambridge CB2 4AT, UK
}




\begin{abstract}
A correlation multi-variate analysis of variance (MANOVA) test to statistically analyze changing patterns of multi-electrode array (MEA) electrophysiology data is developed. The approach enables us not only to detect significant mean changes, but also significant correlation changes in response to external stimuli. Furthermore a method to single out hot-spot variables in the MEA data both for the mean and correlation is provided. Our methods have been validated using both simulated spike data and recordings from sheep inferotemporal cortex.
\end{abstract}




\section{Introduction}

The neural activities induced by a stimulus, such as an odor, an image or a sound, are routinely recorded by multi-electrode arrays (MEA, see Victor, 1994; Gross, 1995; Rutten et al., 2001; Feng, 2004). Usually hundreds or even thousands of local field potentials and neuronal activities are simultaneously recorded at a sample frequency between $1000 \mathrm{~Hz}$ and $2000 \mathrm{~Hz}$ (Mehring et al, 2003; Todd, 2005). Such an experiment could last a few hours and produce data sets of hundreds of GBytes. Several analytical software packages(e.g. Neuroexplorer, MATLAB) are available for editing and visualizing data, and involving in time-frequency analysis(Masimore, 2004). However, there is very little that has been proposed for detailed statistical analysis and subsequent visual representations of changing spatial and temporal patterns of information recorded across an electrode array. Although many methods have been proposed to analyze single cell activity, such techniques are not directly applicable to multi-neurons activities (Shaw,1999; Horton et al. 2005).

The first step of analyzing MEA data, in particular, the local field potential data and spike trains, is to single out which variables (neurons or electrodes) are sensitive or responsive to the stimulus(odor, image or sound). For such a task, multivariate analysis of variance (MANOVA)(Johnson,2002) is probably the first choice to test the difference between two populations with spatial and temporal patterns. When we apply MANOVA to MEA data, we have demonstrated its pros and cons in Horton et al. (2005). A modified version of MANOVA, which we call adaptive MANOVA (Wu et al. 2006), has been proposed to deal with MEA data sets. We have shown that the adaptive MANOVA can not only perform the statistical testing task, but also accurately detect the hot-spot variables, i.e., variables respond to an external stimulus. 
However, adaptive MANOVA, as in the traditional MANOVA, is designed to test the changes in mean activities. As we all know, the mean activity of a neuronal network could remain unchanged when a stimulus is presented, but it could somehow reorganize itself to show more or less coherent activities. A typical example is that two neurons fire independently, but synchronize themselves whenever a stimulus is applied. The mean firing rates before and during stimulus are identical. The question we ask here is whether adaptive MANOVA could detect the coherence (correlation) changes in the recorded physiological data. Here coherence changes imply that the covariance matrix between variables before and during stimulus is different. Pattern changes include both mean activity (mean vector) and coherent activity (covariance matrix) changes.

A prerequisite for the applicability of adaptive MANOVA is that the covariance matrices among variables are equal. The fact is that for local field potentials and spike trains data, the correlations between variables are likely to change due to the stimulus, as mentioned above. Hence the adaptive MANOVA is not applicable when the covariance matrices vary during the stimulus.

In this paper, we propose methods to test pattern changes in physiological data, especially MEA data including both local field potentials and spike trains. We extend adaptive MANOVA to detect the correlation matrix changes in response to an external stimulus. In doing this, the Fisher's $z$ transformation and Edgeworth expansion(Bhattachary and Ghosh,1978; Blinnikov and Moessner, 1998) are introduced. The transformation helps us to transform the distribution of covariance, which is not Gaussian in general, into Gaussian distribution, which is required in a MANOVA analysis. The expansion provides us with a criterion to assess how close is the joint distribution of transformed covariance and a multivariate normal distribution. After overcoming these difficulties, 
the adaptive MANOVA can be directly applied to test the changes of the covariance matrix. The detected hot-spot correlations reveal the changed pair-variables in respondence to the stimulus.

The method is first applied to two types of simulated spike data, which demonstrates the necessity of correlation MANOVA test. We then apply the method to the local field potentials recorded while animals performed an operant discrimination task between different pairs of sheep faces in order to obtain a food reward. The paper first reviews the mean MANOVA test, and then develops correlation MANOVA test based on it. An algorithm for correlation MANOVA test is proposed in method section (Section 2). A full version of MANOVA is introduced in Section 2 as well. Our approach is then tested by using simulated spike data and recordings from sheep's vision experiments.

\section{Methods}

Traditional multi-variate analysis of variance(MANOVA) is used to investigate whether two mean vectors are significantly different or not (Johnson, 2002). In dealing with physiological data MANOVA could be extremely useful when the dimension of the population vectors is small. But as we have mentioned above, for MEA data we usually face a huge amount of data with a large dimensionality (i.e., a dimension $>1000$ ). MANOVA in its textbook version is proved not to be the ideal choice to handle high dimensional population dataset, especially for complex biological data such as LFPs(local field potentials) recorded from MEA, Gene Microarrays, multi Spike trains and fMRI data(functional magnetic resonance imaging) (Horton et al., 2005). Observing 
from electrophysiological recordings, it is possible that information seems to be carried by a small number of significant channels or neurons rather than the whole set of channels or neurons. The difficulty we are facing is how to pick up the hot-spots(significantly changed variables) from the whole group of variables. An adaptive MANOVA and a corresponding HOTTOR algorithm to resolve the issue has been proposed in Wu et al. (2006), and the method is proved to be very useful in detecting the hot-spots. But a restriction in the paper is that the correlation matrix of the variables of pre-stimulus and during-stimulus must be identical.

Fig. 1 demonstrates the situation with mean changes (left) and variance changes (right). When the covariance is identical for pre- and during-stimulus, the mean MANOVA test could be successfully applied to test the difference. When the covariance is different, mean MANOVA test is no long applicable no matter whether the mean values are the same or not. Fig. 1 (right) shows that the covariance of variables is significantly changed but the mean values are identical. As we all know, synchronization between neurons could play a significant role in information processing (Lee, 2004) and a proper statistical approach to test and single out changed pair-neurons is of vital importance.

In this section we will first introduce the adaptive MANOVA, which we call mean MANOVA test. Then, we will discuss the method to test the changes of the correlation matrix of recorded variables, which is termed correlation MANOVA test. An algorithm (HOTTOR) to pick up changed variables is proposed. Finally, a full version of correlation MANOVA is introduced. 

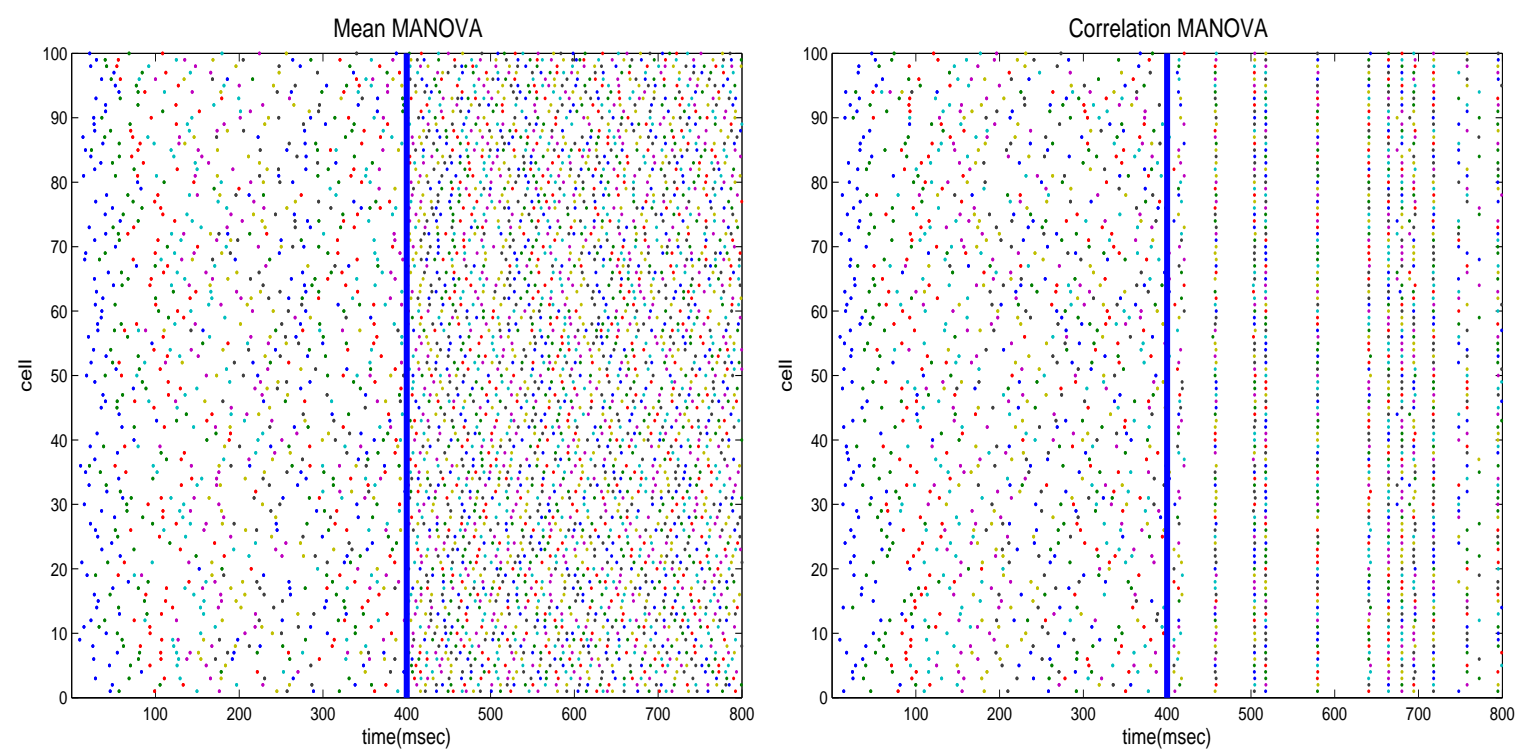

Figure 1: Raster plot of 100 neuron activity generated from the integrate-and-fire models. Left, the mean firing rate of neurons in the left and right hand of the vertical thick line is different, i.e., at around $400 \mathrm{msec}$, a stimulus is turned on (a constant current is added in the model). Right, the mean firing rate of neurons in the left and right hand of the vertical thick line is identical, but their correlation changes, i.e., at around 400 msec, a stimulus is turned on (inputs are correlated). 


\subsection{Models}

Let $X_{1}=\left(x_{11}, \cdots, x_{1 m}\right)$ (say, pre-stimulus LFPs), $X_{2}=\left(x_{21}, \cdots, x_{2 m}\right)$ (say, during-stimulus LFPs) be two random matrices. The spatial and temporal pattern of the recordings can be represented by the rows and columns of the matrices. For example, for LFP data, $x_{1 i}$ is the one pre-stimulus LFP variable recorded from time $[0, N h]$, where $h$ is the sampling resolution and $N$ is the total sample size. Correspondingly we have

$$
\Phi_{1}=\left\{\phi_{1 i j}=\left(x_{1 i}-E x_{1 i}\right)\left(x_{1 j}-E x_{1 j}\right)\right\}_{i, j=1, \cdots, m}
$$

and

$$
\Phi_{2}=\left\{\phi_{2 i j}=\left(x_{2 i}-E x_{2 i}\right)\left(x_{2 j}-E x_{2 j}\right)\right\}_{i, j=1, \cdots, m}
$$

the random covariance matrix of $X_{1}$ and $X_{2}$.

The mean MANOVA test is designed to find all variables between $X_{1}$ and $X_{2}$, which are significantly different. In fact, a pre-requirement to perform the mean MANOVA test is that

$$
E \Phi_{1}=E \Phi_{2}
$$

In other words, before carrying out a mean MANOVA test, we have to test whether Eq. (2.1) is true or not, which is the main concern of the current paper.

In Fig. 1, raster plot of 100 neuron activities is presented. Spikes are generated from the integrate-and-fire model with independent or correlated Poisson inputs. For spikes data and for a given time window $\left[k T_{w},(k+1) T_{w}\right] \subset[0, T]$, where $T_{w}$ is the recording time window, define

$$
x_{i k}=\frac{N_{\left[k T_{w},(k+1) T_{w}\right]}}{T_{w}}, i=1,2
$$


where $N_{\left[k T_{w},(k+1) T_{w}\right]}$ is the number of spikes recorded in the time window, $k$ ranges from 0 to $T / T_{w}-1$, and $T$ is the total length of recordings. It is known that for $i=1,2$ the joint distribution of $X_{i}$ is normal(Johnson,2002). For example, in both figures in Fig. 1, the spikes in the left hand side of the thick vertical line could be spontaneous activity (pre-stimulus), which yields $X_{1}$. The right hand side of the thick vertical line gives us $X_{2}$. The data in Fig. 1 is generated according to the fact that $E X_{1} \neq E X_{2}$, but $E \Phi_{1}=E \Phi_{2}$ (left) and $E X_{1}=E X_{2}$, but $E \Phi_{1} \neq E \Phi_{2}$ (right). Mean MANOVA can handle the situation in Fig. 1 (left) but not in Fig. 1 (right).

\subsection{Mean MANOVA test}

For the completeness of our current paper, we first present a brief review of the mean MANOVA. For population 1 , let us assume that the first $n$ variables have mean 1 , and the rest have mean 0 . For population 2, the first $n$ variables have means $c_{1}, c_{2}, \cdots, c_{n}$ respectively, and the rest have means 0 . In other words, $n$ random variables take different values due to, say, different treatments or stimuli. The hot-spot is $\{1,2, \cdots, n\}$. In the literature, the most common way to compare the difference between two populations is MANOVA. In MANOVA, we first construct a statistical quantity called Wilks lambda. The validity of the hypothesis that $\vec{\mu}_{1}:=E X_{1}$ (the mean activity of population 1 ) $=\vec{\mu}_{2}:=E X_{2}$ (the mean activity of population 2) or not is assessed based on Wilks lambda.

In order to evaluate the Wilks lambda, we need the covariance matrices of both populations. In this section, the covariance matrix $\Sigma=E \Phi_{1}=E \Phi_{2}$ is assumed to be equal and generated randomly(each upper triangle element is i.i.d. $N\left(0, \sigma^{2}\right)$, since the covariance matrix must be symmetric and positive definite). 
MANOVA is defined based on the Wilks lambda $\Lambda$,as in Horton(2005). The significance score of $A=\{1, \cdots, m\}$ is given by

$$
S(A)=-\left[N-\frac{m+2}{2}\right] \log (\Lambda(A)) / \chi_{m}^{2}(\alpha)
$$

where $\chi_{m}^{2}(\alpha)$ is the upper $(100 \alpha)$ th percentile of a chi-square distribution with $m$ degrees. The element of set $A$ is the index of the variable, i.g., the $i$ th element of $A$ stands for the $i$ th variable for both populations. $\Lambda(A)$ is computed according to appendix A. For any subset $B \in\{1, \cdots, m\}$, the significance score could be defined accordingly. Obviously, a subset $B$ is significantly changed if its score $S(B)$ is larger than unity.

By defining the significance score, a few interesting properties are found for the significance score. First of all, the significance score is a decreasing function of $m$ when $m>n$. Secondly the significance score is an increasing function of $n$ when $m$ is fixed. Thirdly, the significance score is a decreasing function with respect to $\rho(\rho$ is the correlation coefficient between variables) with all other parameters fixed. Fourthly, the derivative of significance score with respect to a subset $B=\{1, \cdots, i\}$ is not continuous at $i=n$. It is the crucial property to develop the following HOTTOR algorithm to detect the hot-spot.

Fig 2 presents a simple simulated example to demonstrate the properties we discussed above. In this example, we assume all $c_{i}$ s equal to $c$ for simplicity and the covariance matrix is 


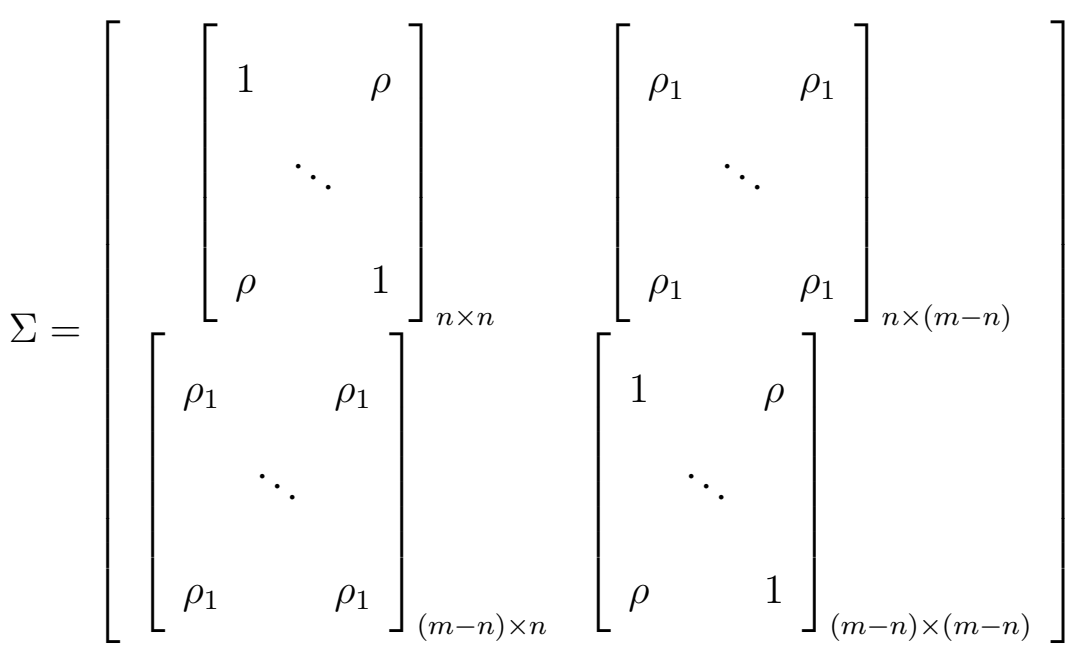

where we assume that $\left|\rho_{1}\right|<<|\rho|$, reflecting the structure in means. There are total 100 variables for each population, and only first 30 have significant change from population 1 to population 2 . The sample size is 200 .

Three dimensional significance score is plotted against the subset $A$ and values of $c \in[0,1]$ for three different cases, $\rho<0, \rho=0, \rho>0$. Obviously, for fixed subset $A$ and $c$, the interesting point here is that the significance score for $\rho<0$ is always larger than that for $\rho>0$. This agrees with one of our long-term working hypotheses: it is much easier to detect the changes in a system with negatively correlated units than with positively correlated or independent units.

The two dimensional significance score is also presented with $c$ fixed. The significance score reaches maximum when only part of the significantly changing variables are included. Inflexion occurs when exactly all the significantly changing variables are involved in the test. This means inflexion rather than the maximum significance score can be regarded as the criterion to detect the whole significance variables.

After the significance score is defined and the proper algorithm is employed, the detection of 


\section{3-D Significance score plot}

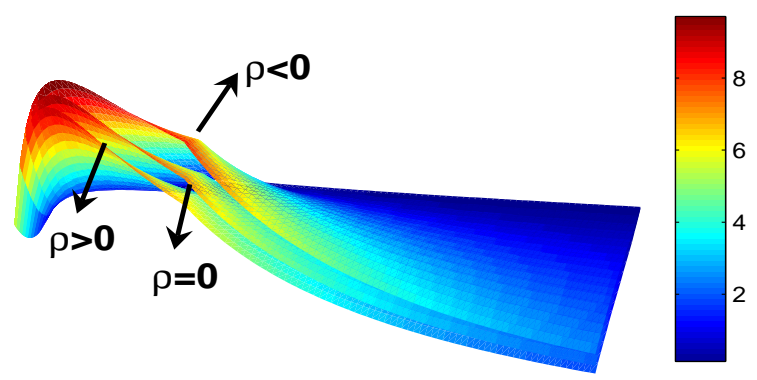

2-D Significance score plot $(\rho>0)$

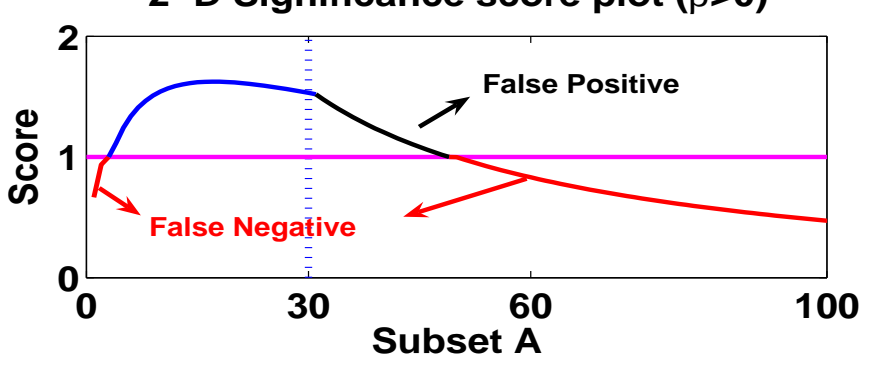

Figure 2: Upper panel: 3-D significance score is plotted against the subset $A$ and $c \in[0,1]$. There is an inflexion point for all three figures in the upper panel, and this point could be identified clearly at the lower panel at point 30. 
the hot-spots is quite routine. But as we mentioned at the beginning of the section, it is a prerequirement to satisfy Eq. (2.1) in mean MANOVA. In the next subsection we turn our attention to the assumption Eq. (2.1), which is of physiologically interests as well, as mentioned before.

\subsection{Correlation MANOVA test}

For two populations, which both follow multi-normal distributions, there only exist the following relations listed below since the distributions are fully determined by their means and variances.

1. Equal means and variances $\Longrightarrow$ Same population.

2. Different means, equal variances $\Longrightarrow$ Mean MANOVA test applicable.

3. Different means, equal variances $\Longrightarrow$ Mean MANOVA test not applicable.

4. Equal means, different variances $\Longrightarrow$ Mean MANOVA test not applicable.

If the variances are different, mean MANOVA test is not applicable. To determine whether the two populations are significantly different or not, we have to compare the covariance matrices $\Phi_{1}$ and $\Phi_{2}$. From Kenney (1962) and Eckhardt (1984), we have the exact form of the distribution of the correlation(Eq. (2.5) and Eq. (2.6)). Actually, we will go even a step further to define

$$
\phi_{k i j}(N)=\frac{\sum_{\xi=1}^{N}\left(x_{k i}(\xi)-E x_{k i}(\xi)\right)\left(x_{k j}(\xi)-E x_{k j}(\xi)\right)}{\sum_{\xi=1}^{N}\left(x_{k i}(\xi)-E x_{k i}(\xi)\right) \sum_{\xi=1}^{N}\left(x_{k j}(\xi)-E x_{k j}(\xi)\right)}
$$

where $x_{k i}(\xi)$ is the $\xi$ th element of the vector $x_{k i}, k=1,2, i<j, i, j=1, \cdots, m$. Since the correlation matrix is symmetric, the number of variables involved is $\frac{m(m-1)}{2}$. 
If $E \phi_{k i j}=0$, the probability density function of $\phi_{k i j}(N)$ is

$$
p(r)=r \sqrt{\frac{\nu}{1-r^{2}}} \sim t-\text { distribution }
$$

$\nu=N-2$.

If $E \phi_{k i j} \neq 0$, let $I=\frac{\nu-2}{2}$ and $J=\frac{\nu-3}{2}$, the probability density function of $\rho$ is

$$
p(r)= \begin{cases}1-\frac{2}{\sqrt{\pi}} \frac{\Gamma((\nu+1) / 2)}{\Gamma(\nu / 2)} \sum_{k=0}^{I}(-1)^{k} \frac{I !}{(I-k) ! k !} \frac{|r|^{2 k+1}}{2 k+1}, & \text { for } \nu \text { even } \\ 1-\frac{2}{\sqrt{\pi}}\left[\sin ^{-1}|r|+|r| \sum_{k=0}^{J} \frac{(2 k) ! !}{(2 k+1) ! !}\left(1-r^{2}\right)^{k+1 / 2}\right], & \text { for } \nu \text { odd }\end{cases}
$$

If we define

$$
Z_{k i j}(N)=\tanh ^{-1} \phi_{k i j}(N)
$$

the variable $Z_{k i j}(N)$ is approximately normally distributed with a mean $\mu_{Z}(k, i, j, N)=$ $\tanh ^{-1} E \phi_{k i j}$ and variance $\sigma_{Z}^{2}(k, i, j, N)=\frac{1}{N-3}$. Actually, the transformation defined by Eq. (2.7) is usually called Fisher's z transformation(David, 1949).

Now it is clear that every element of the correlation matrices follows a normal distribution with the same variance if $N$ is kept as a constant. The issue now is whether the joint distribution of

$$
\bar{Z}_{k}(N)=\left\{Z_{k i j}(N)\right\}_{i<j, i, j=1, \cdots, m} \quad k=1,2
$$

still follows a normal distribution. However, it is possible that the joint distribution does not follow a multivariate normal distribution even if every element is normally distributed.

In order to determine whether the joint distribution of the elements follows a normal distribution or not, the Edgeworth expansion of the density $p(\bar{\rho}), \bar{\rho}=\left[\rho_{1}, \ldots, \rho_{d}\right]$ with $d=m(m-1) / 2$, is introduced. Edgeworth expansion approximates a probability distribution in terms of its cumulants and Hermite polynomials. Let $p_{n}$ be the probability density function, and it is chosen to be a 
normal distribution with mean and variance equal to the mean and variance of $\left\{\bar{Z}_{k}(N)\right\}, k=1,2$. Then we can expand the probability density function $p(\bar{\rho})$ to

$$
p(\bar{\rho}) \approx p_{n}(\bar{\rho})\left(1+\frac{1}{3 !} \sum_{\xi, \eta, \zeta} \kappa^{\xi, \eta, \zeta} h_{\xi \eta \zeta}(\bar{\rho})+\frac{1}{4 !} \sum_{\xi, \eta, \zeta, \tau} \kappa^{\xi, \eta, \zeta, \tau} h_{\xi \eta \zeta \tau}(\bar{\rho})+O(\bar{\rho})\right)
$$

with $h_{\xi \eta \zeta}$ being the $\xi \eta \zeta$ th Hermite polynomial, $\xi, \eta, \zeta \in\{1, \ldots, d\}$, and $\kappa^{\xi, \eta, \zeta}$ the corresponding standardized cumulant defined by $\kappa^{\xi, \eta, \zeta}=\frac{\kappa^{\xi \eta \eta \zeta}}{\delta_{\xi} \delta_{\eta} \delta_{\zeta}}$, with $\kappa^{\xi \eta \zeta}$ the cumulants $\xi, \eta, \zeta, \tau \in\{1, \cdots, d\}$ and $\delta_{\zeta}$ is the standard deviation of the $\zeta$ th random variable. Similarly, $h_{\xi, \eta, \zeta, \tau}$ is the $\xi \eta \zeta \tau$ th Hermite polynomial and the corresponding standardized cumulant $\kappa^{\xi, \eta, \zeta, \tau}$ is defined by $\kappa^{\xi, \eta, \zeta, \tau}=\frac{\kappa^{\xi \eta \zeta \tau}}{\delta_{\xi} \delta_{\eta} \delta_{\zeta} \delta_{\tau}}$ with $\kappa^{\xi \eta \zeta \tau}$ the cumulant.

In Eq. (2.9), the probability density function is approximated by a normal distribution plus a residual term, which could be fully determined by the cumulants of the given variables. $p_{n}(\bar{\rho})$ is the main term for the approximation, but the magnitude of the second term is important in determining the approximation accuracy. To estimate the difference between $p(\bar{\rho})$ and its corresponding normal distribution $p_{n}(\bar{\rho})$, let us define

$$
Q=1-\frac{1}{3 !} \sum_{\xi, \eta, \zeta} \kappa^{\xi, \eta, \zeta} \min _{k=1,2} h_{\xi \eta \zeta}\left(\left\{\mu_{Z}(k, i, j, N)\right\}_{i<j, i, j=1, \cdots, m}\right)
$$

as the measurement of the approximation accuracy. In the sequel, if $Q$ is greater than a significant level(by running the simulation, $85 \%$ can be regarded as the significant level), then we can conclude that the joint distribution $p(\bar{\rho})$ approximately follows a multivariate normal distribution.

To determine the magnitude of $\frac{1}{3 !} \sum_{\xi, \eta, \zeta} \kappa^{\xi, \eta, \zeta} h_{\xi \eta \zeta}(\bar{\rho})$, we need some more basic knowledge of cumulant(Kenny 1962; Abramowitz,1972), which is explained in Appendix B. 
The joint distribution of the transformed correlations could be calculated through Eq. (2.9) when the cumulants are estimated from the empirical data. If the joint distribution approximates a multivariate normal distribution well, then Mean MANOVA test is applicable to the correlations.

The procedure for correlation MANOVA model can be as

1. Calculate the correlation matrix $\Phi_{1}$ and $\Phi_{2}$ for population 1 and 2 , respectively.

2. Apply inverse hyperbolic tangent transformation to each $\phi_{k i j}(N)$.

3. Calculate the joint distribution of the transformed correlations, compare the distribution with corresponding multivariate normal distribution(Eq. (2.9), Eq. (2.10)).

4. Apply Mean MANOVA test to the transformed correlation variables.

The key point for correlation MANOVA test is whether the joint distribution of the transformed correlations follows a multivariate normal distribution. As we discussed, every single correlation transformed by Fisher's z transformation approximately follows a Gaussian distribution, but the joint distribution may not follow multivariate normal distribution. The Edgeworth expansion is applied to test whether the joint distribution can be approximated by Gaussian distribution. As mentioned, if the approximation is good enough (more than $85 \%$ similarity), the joint distribution could be regarded as multivariate normal distribution and then the mean MANOVA test is directly applicable to the transformed correlation variables. The application procedure is fully described in the following section. 


\subsection{Algorithm}

The whole algorithm is divided into three parts. The first part is unique for correlation MANOVA test, the other two parts are the same as the mean MANOVA test.

\section{Part I: Transformation part}

First we introduce the notation involved in the algorithm. Suppose we have two populations $X_{1}=\left(x_{11}, x_{12}, \cdots, x_{1 m}\right)$ and $X_{2}=\left(x_{21}, x_{22}, \cdots, x_{2 m}\right)$ with same number of variables $m$ and sample size $N$. The elements $\rho_{k i j}(h)$ of correlation matrix $\Phi_{k}$ are calculated by Eq. (2.11), the dimension of $\Phi_{1}$ is $m \times m \times(N-M+1)$. $M$ is the number of samples employed to calculate the correlation. $h$ increases from 1 to $N-M+1$.

$$
\rho_{k i j}(h)=\frac{\sum_{q=h}^{h+M-1} x_{k i}(q) x_{k j}(q)}{\sum_{q=h}^{h+M-1} x_{k i}(q) \sum_{q=h}^{h+M-1} x_{k j}(q)}
$$

Let $z_{k i j}(h)=\tanh ^{-1} \rho_{k i j}(h)$ (Fisher's z transform). After the transformation, the Edgeworth expansion is employed to measure the similarity of the joint distribution with Gaussian distribution. If the distribution is not multivariate normal distribution, correlation MANOVA test is not applicable (see Discussion). If the distribution is approximately multivariate normal distribution, we define $Y_{k}$ be the collection of all the upper diagonal elements of $\left\{z_{k i j}\right\}$. The dimension of $Y_{k}$ is $\frac{m(m-1)}{2} \times\left(N-N^{\prime}+1\right)$, and can be regarded as two new populations for further analysis.

\section{Part II: Monotonic part}

First we compute the single unit pairs, which produce maximum or minimum significance 
score between population $Y_{1}$ and $Y_{2}$, and let these two pairs be $\left(i_{\min }, j_{\min }\right)$ and $\left(i_{\max }, j_{\max }\right)$. Let $A$ be the whole set containing $\{(i, j)\}$, where $i$ ranges from 1 to $m-1$ and $j$ from 2 to $m, A_{q}$ be the set containing significantly changing variables. The initial value for $A_{q}$ is $\left(i_{\max }, j_{\max }\right)$.

Then selecting a unit pair, say $\left(i_{1}, j_{1}\right)$, from set $A /\left\{\left(i_{\min }, j_{\min }\right),\left(i_{\max }, j_{\max }\right)\right\}$, we calculate $S\left(A_{q} \cup\left\{\left(i_{1}, j_{1}\right)\right\}\right)$ (refer to Eq. (2.2)). If $S\left(\left\{\left(i_{1}, j_{1}\right)\right\}\right)<S\left(A_{q} \cup\left\{\left(i_{1}, j_{1}\right)\right\}\right)$, then $\left(i_{1}, j_{1}\right)$ is one of the changed unit pairs. Continue this procedure and we add the unit pair to $A_{q}$ if the significance score increases and discard the pair if the significance score decreases. The monotonic step stops when the significance score reaches its maximum. This can be achieved by comparing all the significance scores.

\section{Part III: Reference part}

After finding the unit pairs, which positively contribute to the significance score, now we have to deal with those units, which do not positively contribute to the significance score, but they change their mean values nonetheless. At this step, we assume that at least one pair, which does not change its activity is picked up, say $\left(i_{\min }, j_{\min }\right)$. We use the score of this unit as a reference to assess whether a remaining pair, say $(i, j)$, changes its activity or not. Denote the obtained set as $A_{q+} \supset A_{q}$. If it changes, i.e., $S\left(A_{q+} \cup\left\{\left(i_{\text {min }}, j_{\text {min }}\right)\right\}\right)<S\left(A_{q+} \cup\{(i, j)\}\right)$, we then add it to the changed set $A_{q+}=A_{q+} \cup\{(i, j)\}$, and continuous our selections. If it does not change, $S\left(A_{q+} \cup\left\{\left(i_{\text {min }}, j_{\text {min }}\right)\right\}\right) \geq S\left(A_{q+} \cup\{(i, j)\}\right)$, we then simply choose a new pair to check. Continuous the procedure above until we check all units. We then have all the significantly changing pairs $A_{q+}$.

The procedure of the algorithm could be expressed as a flow chart, seen in Fig. 3. The flow 
chart is divided into three parts, which represent the three steps. Each part should be finished first, then next part starts. The red arrow is the only relation between part I and part II, and part II and part III. The meaning of the symbols are explained below:

- $A$ The whole set $\{(i, j)\}, i$ ranges from 1 to $m-1$ and $j$ from 2 to $m$.

- $\left(i_{\min }, j_{\min }\right)$ The unit pair with minimum significance score.

- $\left(i_{\max }, j_{\max }\right)$ The unit pair with maximum significance score.

- $A_{q}$ The set containing indexes of the significantly changing variables.

- $A_{p}$ The set containing indexes of the significantly and insignificantly changing variables.

\subsection{Recording from Inferotemporal Cortex}

Electrophysiological data were acquired from the inferotemporal cortex of awake behaving sheep (Nicol et al. 2005; Tate et al. 2005). All experimental procedures involving animals were conducted in strict accordance with guidance on the operation of the Animals (Scientific Procedures) Act 1986(http://www.archive.office-documents.co.uk/document/hoc/321/321.htm). Sheep, under halothane (fluothane) anaesthesia, were implanted with two chronic 64-channel MEAs in the right and left inferotemporal cortices, respectively. Recordings were made from the unanaesthetised sheep while they performed an operant discrimination task in which different pairs of sheep faces were presented and a correct panel-press response elicited a food reward. Local event-related LFPs are recorded simultaneously from 128 chronically implanted electrodes. LFP time series data are 


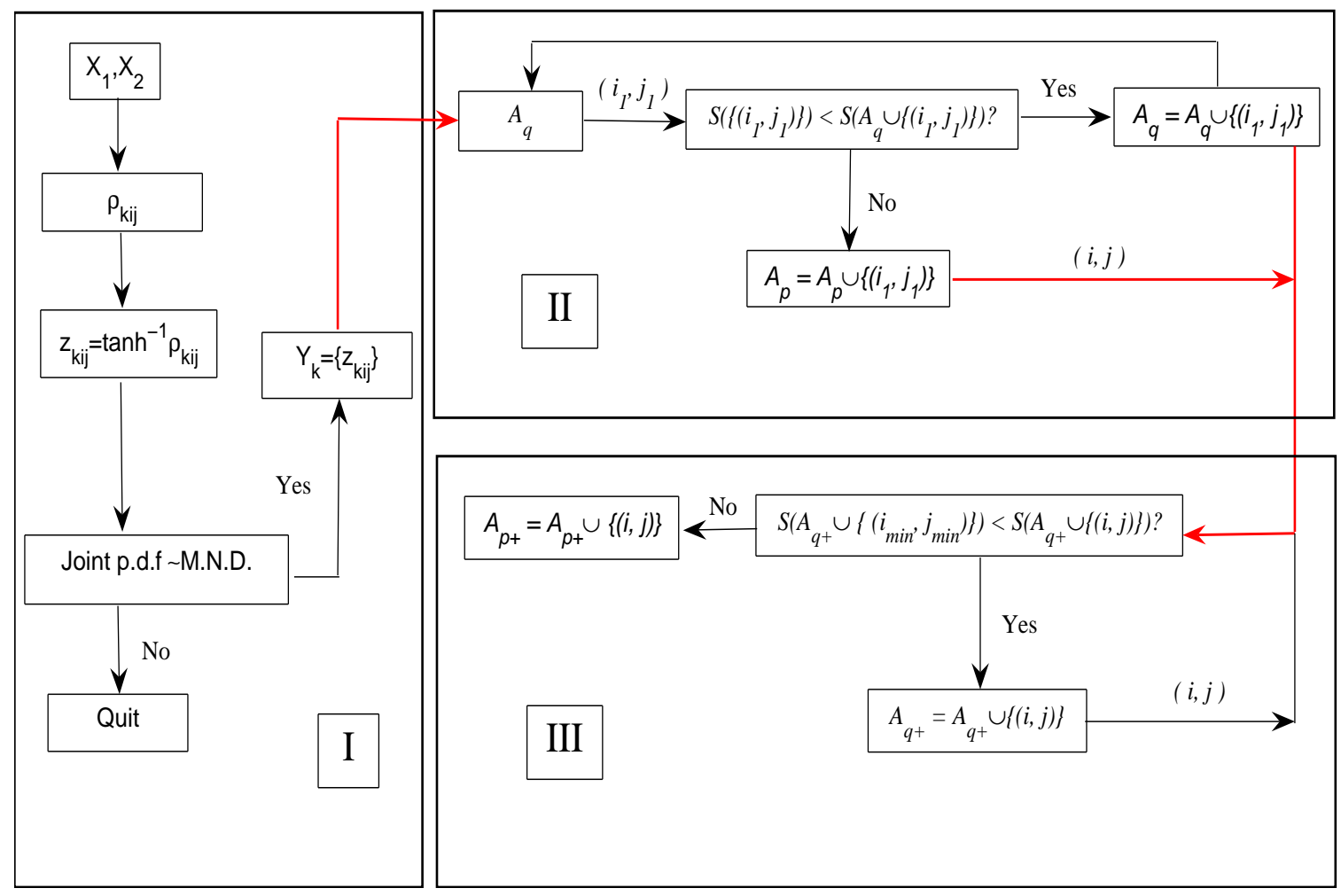

Figure 3: Algorithm flow chart. The transformation part will produce two new populations $Y_{1}$ and $Y_{2}$. Part II and part III are processed based on these two populations. The initial values for $A_{q}$ and $A_{p}$ are $\left\{\left(i_{\max }, j_{\max }\right)\right\}$ and $\left\{\left(i_{\min }, j_{\min }\right)\right\}$, respectively. The initial values for $A_{q+}$ and $A_{p+}$ are the final values of $A_{q}$ and $A_{p}$, respectively. $\left(i_{1}, j_{1}\right)$ and $(i, j)$ are randomly selected from set $A /\left\{\left(i_{\min }, j_{\min }\right),\left(i_{\max }, j_{\max }\right)\right\}$ and $A_{p}$, respectively. 
sampled at 2000 samples/s from around 3 seconds prior to stimulus onset to 3.5 seconds after the stimulus onset in each trial of a session, and stored as 16-bit numbers.

\section{Results}

\subsection{Simulated Spike data}

Before applying correlation MANOVA test to real data, we first test the model by using spike data generated using MATLAB 7.01(The MathWorks, Natick, MA, USA). Two types of spike data are generated. The first type is that the covariance matrix is fixed for pre- and during stimulus, and the mean firing rate for a small number of neurons varies between pre- and during stimulus. Another type is that the mean firing rate is fixed, but the covariance matrix of a small number of neurons varies between pre- and during stimulus.

The raster plot of the data sets is presented in Fig. 4. The data is generated $10^{3}$ seconds before stimulus, and $10^{3}$ seconds during stimulus. In Fig. 4 only 2 seconds data for pre and during stimulus are included. In total there are 300 neurons, but only 10 neurons respond to the stimulus. We randomize the 300 variables such that we can not tell, which variable is responsive to the stimulus. The randomized data set is presented in the Fig. 4 (bottom panel). For the randomized data, the significantly changed neuronal activities are obscured by the large number of unresponsive neuronal activities.

In our analysis, sampling interval $t=100 \mathrm{~ms}$ and total recording time $T=1000 \mathrm{~s}$ are used. There are $N=T / t=10^{4}$ samples for each neurons. The correlation MANOVA test is first 

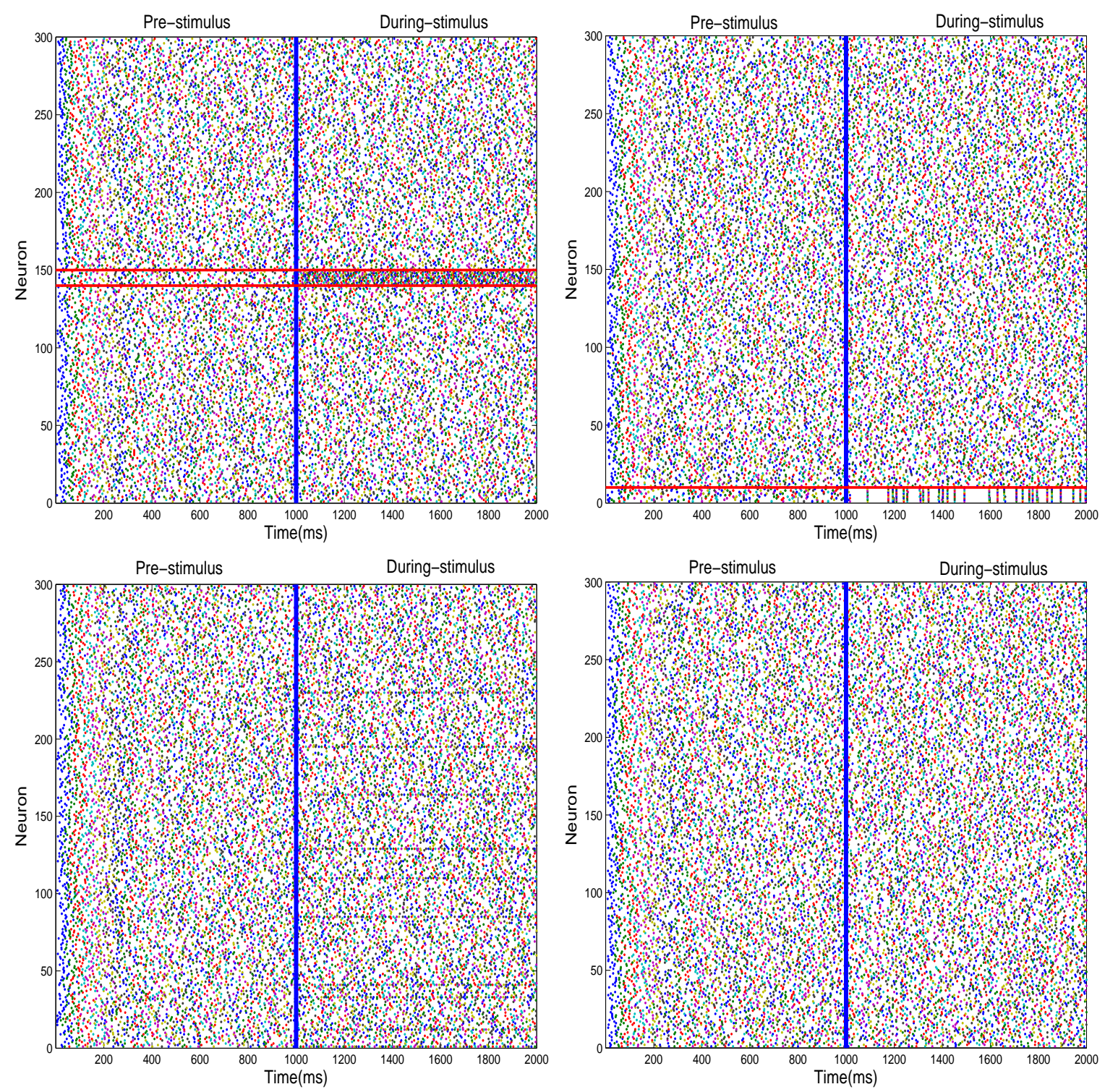

Figure 4: Raster plot of generated data from the integrate-and-fire model. In the upper left panel, a case with different means is illustrated. The covariance between population 1 and population 2 is identical for pre-stimulus and during stimulus (stimulus onset is indicated by the vertical line). The mean firing rate is changed for 10 neurons between pre-stimulus and during-stimulus. In the upper right panel, a case with different covariance is illustrate. The mean firing rates for all variables are the same for pre- and duringstimulus. The covariance matrix between 10 neurons is changed from pre-stimulus to during-stimulus. Bottom panels: identical to the upper panels but neuron labels (from 1 to 300) are randomized. The neurons with significantly changing activities are mixed with other unresponsive neurons. 
applied to the data set on the left hand side of Fig. 4 (left panels). A total $d=\frac{300(300-1)}{2}=$ 44850 correlation variables are calculated for both pre- and during stimulus. When facing such a huge number of variables, we need to divide the variables into groups artificially, and apply mean MANOVA to each divided group. After Fisher's z transformation, ten sets $Y_{1 i}$ and $Y_{2 i}$ are constructed, the dimension for $Y_{1 i}$ and $Y_{2 i}$ is $4485 \times 9000(i=1, \cdots, 10)$, which means that each transformed correlation variable has a sample size of 9000. By applying the mean MANOVA test to $Y_{1 i}$ and $Y_{2 i}$, after 4485 steps selection, no significantly changing variables are detected, which confirms that the correlation matrix does not have any significant change when the stimulus is applied. The mean MANOVA is then directly applied to 300 mean activities. The average firing rates for pre- and during stimulus are presented in Fig. 5 upper panel, and the regions marked red show the significantly changed neurons. All the significantly changing neurons (10 neurons) are detected by mean MANOVA test.

For the data set of Fig. 5 (right upper panel), the average firing rate is a constant. It is not surprising that there is no detected changed activity when mean MANOVA test is directly applied(the maximal significance score is 0.72 ). The correlation MANOVA test is applied to the correlation matrix. There are a total of $d=\frac{300(300-1)}{2}=44850$ correlation variables. These variables are again divided into ten groups. After performing Fisher's z transformation, we have ten sets of $Y_{3 i}$ and $Y_{4 i}$, all with a dimension of $4485 \times 9000$. Then mean MANOVA test is employed to detect the significantly changed variables in each groups. Only 2500 correlations among the total of 44850 variables are depicted in Fig. 5 bottom panel (left and right). The red regions in right bottom panel are the variables that have significantly changed activities. All the changed correlations are fully 
Average firing rate for neurons, pre-stimulus

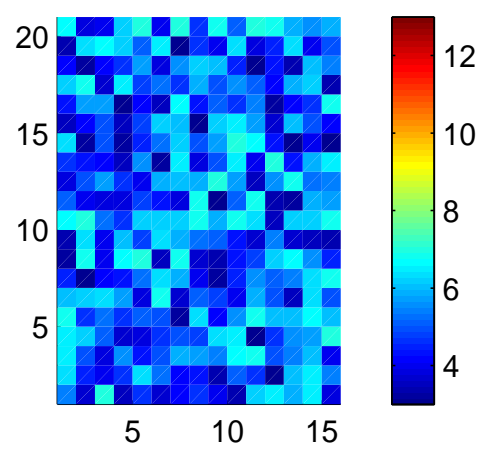

Correlations for pre-stimulus

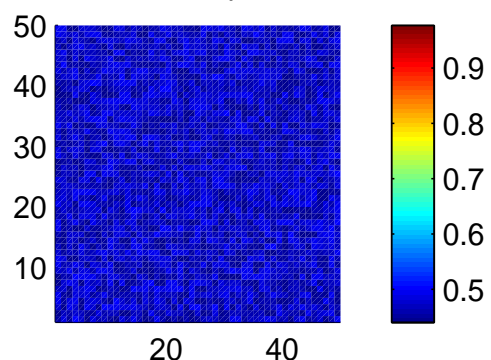

Average firing rate for neurons, during-stimulus

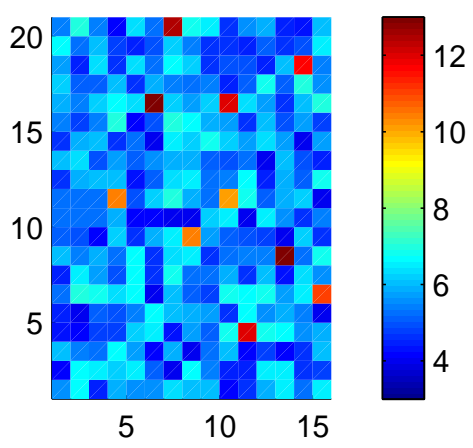

Correlations for during-stimulus

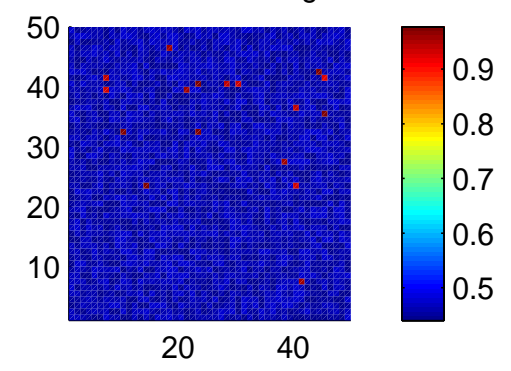

Figure 5: Results of mean MANOVA and correlation MANOVA. Upper panels: Left hand panel depicts average firing rate for pre-stimulus; right hand panel presents average firing rate for during-stimulus. The red regions indicate neurons with significantly changing activities. Bottom panels: left hand panel is the average correlation for pre-stimulus; right hand panel shows average correlation for during-stimulus. The red regions indicate neurons with significantly changing activities. 
detected by our correlation MANOVA.

\subsection{Application to Recordings}

Translating the experiment results into our setup, we have collected data recorded from the left inferotemporal cortex: $X_{1}=\left(x_{1,1}, x_{1,2}, \cdots, x_{1,64}\right)$ pre-stimulus, and $X_{2}=\left(x_{2,1}, x_{2,2}, \cdots, x_{2,64}\right)$ during-stimulus. For $x_{i, j}, i$ is referred to pre- or during-stimulus, $\mathrm{j}$ is the channel number. The similar data have been recorded from the right inferotemporal cortex. As discussed at the beginning of the paper, the issue we face now is to pick up changed variables from the large experimental data set.

Before applying the algorithm, pre-processing the data is necessary. The data is standardized: each variable is divided by its square root of variance. As before, $\Phi_{1}$ and $\Phi_{2}$ are the sample correlation matrix with dimension $64 \times 64 \times 6000$. Fisher's $\mathrm{z}$ transformation is applied to the sample correlation matrix. Then by employing Edgeworth expansion, the similarity of transformed joint distribution of the correlation matrix to a multi-normal distribution is calculated. The similarity factor for pre-stimulus and during-stimulus are $88.46 \%$ and $93.28 \%$ respectively, which imply that the joint distribution can be regarded as a multivariate normal distribution and mean MANOVA test is applicable to the covariance matrix. By applying the algorithm to the transformed correlation matrices, the significantly changed correlations are detected and presented in Fig. 6. The circle is the template of the multi-electrode array, and each number represents one electrode. The red numbers represent the variables, which are responsive to the stimulus. Fig. 6 presents the results of the correlations for both left and right cortex. The number of significantly changed variables 
detected by correlation MANOVA test is 10 and 14 out of 64 channels for the left and right cortex respectively.

The channels with a significantly changed correlations are connected by lines in Fig. 6 . The green lines indicate that the correlation increases during the stimulus, but the blue lines imply the reduction of the correlation during the stimulus. The values of correlations for pre-stimulus and during-stimulus between these variables are further listed in table 1 and table 2. Among changed channels, correlations increase more than decrease. The increased correlations tell us that more regions are coherently more active or less active during stimulus.

Table 1. Correlation list for left visual cortex

\begin{tabular}{|ccc|}
\hline Variable pair & Correlation(Pre-stimulus) & Correlation(During-stimulus) \\
\hline 20,27 & 0.34 & 0.58 \\
\hline 27,34 & 0.24 & 0.49 \\
\hline 22,29 & 0.13 & 0.42 \\
\hline 20,36 & -0.04 & 0.51 \\
\hline 38,45 & 0.31 & -0.10 \\
\hline 52,55 & 0.11 & 0.45 \\
\hline
\end{tabular}

Table 2. Correlation list for right visual cortex 


\begin{tabular}{|ccc|}
\hline Variable pair & Correlation(Pre-stimulus) & Correlation(During-stimulus) \\
\hline 12,19 & 0.21 & 0.52 \\
\hline 12,21 & -0.12 & 0.31 \\
\hline 21,29 & 0.63 & 0.18 \\
\hline 23,39 & 0.02 & 0.47 \\
\hline 27,36 & 0.18 & 0.59 \\
\hline 27,34 & -0.07 & -0.42 \\
\hline 38,39 & 0.24 & 0.56 \\
\hline 46,53 & 0.41 & -0.00 \\
\hline 53,59 & 0.08 & 0.38 \\
\hline
\end{tabular}

\section{Discussion}

We have presented a novel approach: correlation MANOVA test based upon our previous work (Horton, 2005; Wu et al. 2006), to analyze the electrophysiological data recorded by multielectrode arrays. By applying the method to both simulated and biological data(spikes and LFPs), we conclude that the approach is successful in detecting variables, which are statistically significantly responsive to an external stimulus. As mentioned before, we face increasingly huge amounts of data in electro-physiological experiments and our method could play a crucial role in tackling such data sets. 

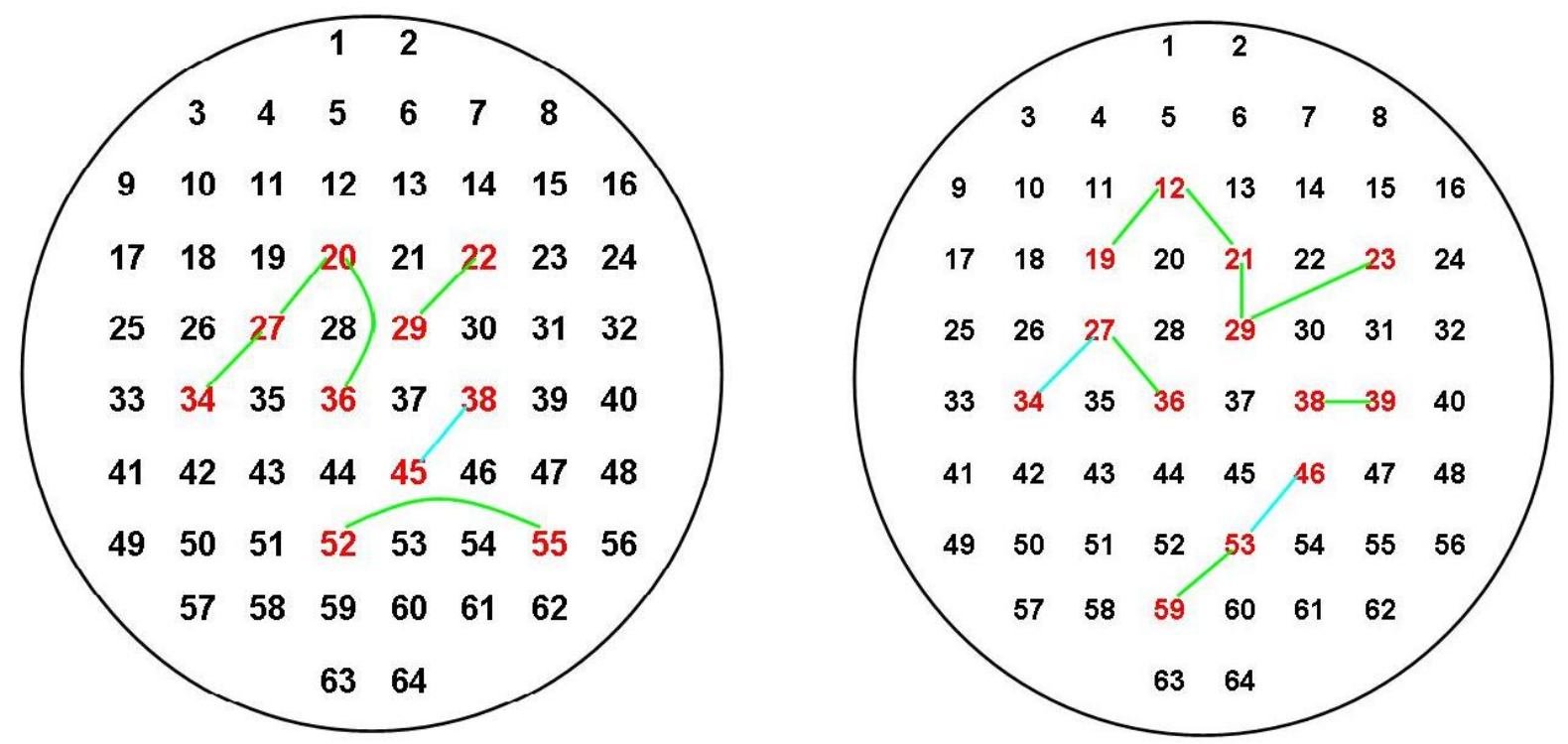

Figure 6: Results of correlation MANOVA test. The green connection lines between variables show that the correlation between channels increase during the stimulus, but the blue lines indicate the decrease of the correlation during the stimulus. The values of correlations among significantly changed channels are listed in table 1 and table 2. 


\subsection{Correlation detection vs. synchronization}

The proposed methods can successfully detect the pattern changes in response to the stimulus, and they also pick up the variables that are statistically significantly responsive to the stimulus. We have demonstrated that the correlation MANOVA test can detect synchronization or de-synchronization between variables. The test detects pair-variables (neurons) that have significantly changed during stimulus. If positive correlation between two variables increases, we could conclude that the variables during stimulus are more synchronized than pre-stimulus.

Of course correlation is a more general notation than synchronization. For example, if we assume that two spike trains are (perfectly) synchronized at spike to spike level, the correlation between the two spike trains is unity. If, in statistical sense, two out of ten spikes are synchronized, then the correlation between the two spike trains is around $2 / 10=0.2$. We could say that the two spike trains are partially synchronized. If the second spike train is delayed by a fraction of the sampling interval, then the two spike trains are no longer (perfectly) synchronized. However, the correlation between two spike trains is unchanged. Hence the detection of correlation changes could be more meaningful than the detection of synchronization, which is more appealing to a visual inspection.

Furthermore, spike trains could exhibit negative correlations, which is beyond desynchronization. Roughly speaking, two variables are negatively correlated implies that if one fires faster, the other should be slower and vice visa. For two periodic signals, a negative correlation between them means there is a phase shift between them. When two periodic signals are negatively correlated with a correlation coefficient of -1 , we know that they are in opposite phases. In terms of 
correlation MANOVA we are able to single out both negative (anti-phase) and positive (on-phase) changes in the recorded signals (neurons).

\subsection{Instantaneous firing rates vs. interspike intervals}

When dealing with spike trains, the results presented in the current paper are based upon the information of instantaneous firing rates. One might argue that it is not the optimal way to tackle the spike train data since interspike intervals might provide more information than instantaneous firing rates. Hence a natural question is whether we could apply our approach to interspike intervals. The answer is confirmative, but it requires some more developments. Essentially, as we have emphasized in the paper,it is a prerequisite for applying MANOVA that the variables are normally distributed. With interspike intervals, we know that they are not normally distributed and, even worse, the exact distribution is unknown. Fortunately, in the past few years, we have successfully developed a non-parametric approach to accurately fit interspike interval distributions (Rossoni and Feng 2005). Therefore, a combination of the results in the current paper (mean MANOVA and correlation MANOVA) and the results in Rossoni and Feng (2005) could lead to a successful application of the hierarchical MANOVA to interspike interval data.

\subsection{Correlation MANOVA and causality}

Due to the correlations (interactions) between variables, multi-variable analysis is the only appropriate way to deal with multi-variable recordings such as multi-electrode array data. Nevertheless, with multi-variables, usually we face the interactions between two or more groups of variables. 
An appropriate tool is required to statistically assess the changes between groups of variables. Correlation MANOVA as we briefly summarized in the current paper serves this role.

In our approach, we consider the interaction between two groups of variables. The interaction (correction) is no-directional. An observation that the activity of neuron B is correlated with that of neuron $\mathrm{C}$ could mean that the input of neuron $\mathrm{B}$ comes from the output of neuron $\mathrm{C}$, or that both of them receive a common input. To distinguish between these cases, we have to take into account the causality analysis(Chen et al., 2004, Albo et al., 2004). Again, causality analysis is usually performed on single to single variable bases and a multi-variable causality analysis is still lacking.

Acknowledgment. J.F. was partially supported by grants from UK EPSRC (EP/C51338X), (EP/D051916), and (GR/S30443).

\section{References}

Abramowitz M, Stegun IA, editors. Handbook of Mathematical Functions with Formulas, Graphs, and Mathematical Tables, 9th ed. Dover; 1972.

Albo Z, Di Prisco GV, Chen Y, Rangarajan G, Truccolo W, Feng J, Vertes R, Ding M. Is partial coherence a viable technique for identifying generators of neural oscillations? Bio. Cyber., 2004;90:318-26.

Bhattachary RN, Ghosh JK. On the validity of the formal Edgeworth expansion. Ann. Stat., 1978;6:434-51.

Blinnikov S, Moessner R. Expansions for nearly Gaussian distributions. Astron. Astrophys. 
Suppl. Ser., 1998;130:193-205

Chen Y, Rangarajan G, Feng J, Ding M. Analyzing multiple nonlinear time series with extended Granger causality. Phys. Lett. A, 2004;324:26-35.

David, FN. The Moments of the $\mathrm{z}$ and F Distributions. Biometrika., 1949;36:394-403

Eckhardt DH. Correlations between global features of terrestrial fields. Math. Geol., 1984;16:155-71.

Feng JF, editor. Computational Neuroscience: A Comprehensive Approach. Chapman and Hall/CRC Press; 2004.

Gross GW, Rhoades BK, Azzazy HM, Wu M-C. The use of neuronal networks on multielectrode arrays as biosensors Biosens. Bioelectron., 1995;10:553-67

Horton P, Bonny L, Nicol A, Kendrick K, and Feng JF. Applications of multi-variate analysis of variance (MANOVA) to multi-electrode array electrophysiology data. J. Neurosci. Methods, 2005;146: 22-41.

Johnson RA, Wichern DW. Applied Multivariate Statistical Analysis, 5th ed. Prentice Hall; 2002.

Kenny JF, Keeping ES. Mathematics of Statistics. 3rd ed. New Jersey: Van Nostrand; 1962: 71-82.

Masimore B, Kakalios J, Redish AD. Measuring fundamental frequencies in local field potentials. J. Neurosci. Methods, 2004;138:97-105

Mehring C, Rickert J, Vaadia E, Cardoso de Oliveira S, Aertsen A, Rotter S. Inference of hand movements from local field potentials in monkey motor cortex. Nature Neurosci., 
2003;6:1253-1254

Nicol AU, Magnusson MS, Segonds-Pichon A, Tate A, Feng J, Kendrick KM. Local and global encoding of odor stimuli by olfactory bulb neural networks. Program No. 476.62005 Abstract Viever/Itinerary Planner. Washington, DC: Society for Neuroscience, 2005. Online. http://sfn.scholarone.com/itin2005/index.html

Rossoni E, Feng J. Non-parametric approach to extract information from interspike intervals. J. Neurosci. Methods, 2005; 150: 30-40.

Rutten W, Mouveroux J, Buitenweg J, heida C, Ruardij T, Marani E ,Lakke E. Neuroelectronic interfacing with cultured multielectrode arrays toward a cultured probe. Proc. IEEE, 2001;89:1013C29

Shaw F-Z, Chen R-F, Tsao H-W, Yen C-T. A multichannel system for recording and analysis of cortical field potentials in freely moving rats. J. Neurosci. Methods, 1999;88:33-43.

Tate A, Nicol A, Fischer H, Segonds-Pichon A, Feng J, Magnusson M, Kendrick K. Lateralised local and global encoding of face stimuli by neural networks in the temporal cortex. Program No.362.1. 2005 Abstract Viewer/Itinerary Planner. Washington, DC: Society for Neuroscience, 2005. Online.

Todd C, editor. Event-related potentials : a methods handbook. MIT Press; 2005.

Van Hulle MM. Edgeworth approximation of Multivariate Differential Entropy. Neural Computation, 2005; 17:1903-10

Victor JD, Purpura K, Katz E, Mao B. Population encoding of spatial frequency, orientation, and color in macaque V1. J. Neurophysiol., 1994;72:2151-66 
Wu JH, Kendrick K, and Feng JF. (2006) Detecting hot-spot in spatio-temporal patterns of complex biological data. Online Source, http://www.dcs.warwick.ac.uk/ jianhua/doc/hottor.html.

\section{Appendix A}

Define $\bar{x}_{l}=\sum_{j=1}^{N} x_{l j} / N$ for $l=1,2$ and $\bar{x}=\sum_{l=1}^{2} \sum_{j=1}^{N} x_{l j} /(2 N)$, where $N$ is the number of samplings. Furthermore, let us define sampling covariances

$$
\begin{aligned}
S S W & =\sum_{l=1}^{2} \sum_{j=1}^{N}\left(x_{l j}-\bar{x}_{l}\right)\left(x_{l j}-\bar{x}_{l}\right)^{\prime} \\
S S B & =\sum_{l=1}^{2} N\left(\bar{x}_{l}-\bar{x}\right)\left(\bar{x}_{l}-\bar{x}\right)^{\prime}
\end{aligned}
$$

Then Wilks' lambda(Johnson,1988) is introduced as:

$$
\begin{aligned}
\Lambda & =\left|\frac{S S W}{S S B+S S W}\right| \\
& =\left|\frac{2(N-1) \Sigma}{S S B+2(N-1) \Sigma}\right| \\
& =\mid \frac{\left[\begin{array}{cc}
\Sigma_{11} & \Sigma_{12} \\
\Sigma_{21} & \Sigma_{22}
\end{array}\right]}{\mid\left[\begin{array}{cc}
\Sigma_{11}+B & \Sigma_{12} \\
\Sigma_{21} & \Sigma_{22}
\end{array}\right]}
\end{aligned}
$$


Where $\Sigma=\left[\begin{array}{cc}\Sigma_{11} & \Sigma_{12} \\ \Sigma_{21} & \Sigma_{22}\end{array}\right]$ and $B=S S B / 2(N-1)$.

\section{Appendix B}

In probability theory and statistics, the cumulant $\kappa_{n}$ of the probability distribution of a random variable $X$ are given by(Kenney,1962)

$$
E\left(e^{t X}\right)=\exp \left(\sum_{n=1}^{\infty} \kappa_{n} t^{n} / n !\right)
$$

The correlation between cumulants and moments gives us an easy way to calculate cumulants from known moments. Cumulants are related to the moments by the following recursion formula:

$$
\kappa_{n}=\mu_{n}^{\prime}-\sum_{k=1}^{n-1}\left(\begin{array}{c}
n-1 \\
k-1
\end{array}\right) \kappa_{k} \mu_{n-k}^{\prime}
$$

The joint cumulant of several random variables $X_{1}, \ldots, X_{n}$ is

$$
\kappa\left(X_{1}, \ldots, X_{n}\right)=\sum_{\pi} \prod_{B \in \pi}(|B|-1) !(-1)^{|B|-1} E\left(\prod_{i \in B} X_{i}\right)
$$

Where $\pi$ runs through the list of all partitions of $\{1,2, \ldots, n\}$, and $B$ runs through the list of all blocks of the partition $\pi,|B|$ is the size of the set $B$. 